\title{
Modern Learning and Training Tools which can be Properly Adapted and Designed to Reinforce Specialized Courses on Effective Crisis Management
}

\author{
Georgios C. Baltos
}

\author{
Ph.D.(c) of Business School, \\ University of the Aegean, Chios, Greece
}

Filippa S. Chomata

M.Sc. on Financial Analysis for Executives, University of Piraeus, Piraeus, Greece

loannis G. Vidakis

Ph.D. of Business School, University of the Aegean, Chios, Greece

Janis Balodis

MA student, Belarusian Economic Research and Outreach Center (BEROC), Minsk, Belarus

Doi: 10.2478/jesr-2018-0028

\begin{abstract}
This paper highlights the effectiveness of several modern training tools that could be applied within the context of training organizations, aiming towards the introduction of theoretical and practical fields, like the assessment of critical risks, the impact of time pressure and the importance of situational awareness, into the contents of a training course. The first part deals with present training tendencies and tools like distance and technology-based learning, while it is highlighted that knowledge management has gone through a deep and wide re-orientation. Quality assurance issues regarding education are also of great significance. The second part then studies the psychology of serving in combat or irregular crisis situations. It is about the particularities that compose the military, business or civil protection reality, resulting in new approaches of training courses that pursue excellent performance, particularly in dynamic and changing with high-velocity environments. Concepts like shared-ness of information, maturity in leadership decision making and cognitive task analysis can all determine a new domain of acceptable measures for crisis management training at professional level. The scope ranges from a routine organizational context to rapid response in a larger-scale emergency under pressure, requiring respective training adjustments.
\end{abstract}

Keywords: Training Tools, Critical Risks, Quality assurance, Situational Awareness, Crisis Management

\section{Introduction}

This study focuses on the analysis of several modern training tools that could be used within the context of civil protection institutions, military academies and training centers, taking into account 
the differences between training for operations under normal circumstances and training specialized in the treatment of emergency situations. The purpose of this discussion is to highlight how the crisis managers or the respective training planners could familiarize themselves with and take advantage of training topics like risk identification and assessment, simulation exercises and intuitive leadership. Such training applies to a wide range of activities including military and civil protection, disaster recovery and plenty of professional case studies concerning both routine organizational contexts as well as rapid responses in larger-scale emergencies. The quantity and quality of the training directly affects the success of the respective operations, adding value to students and professionals involved, but also reinforcing security, stability and safety for the societies in favor of the citizens and their well-being.

\section{Research Horizons for Training Projects}

\subsection{Distance and technology-based learning}

One of the milestones of the new training tendencies over the recent years is distance training. This system is absolutely focused on learning and/or teaching remotely. The relevant old fashion ways were involving the in-person training, while the new training ways activate the two and/or multiple way system of communication. Another qualitative difference is that distance training cannot take place without the assistance of high technology. Any distance training can be actually considered a kind of technologically mediated learning.

Mehdi Khosrow-Pour (Khosrow-Pour 2001) studied the next level of distance training, whereas the students belong in a specific professional environment. Her theory is well known as "corporate distance training", having interesting results in combining organizational cultures with particularly needed distance training features. The key concept of "corporate distance training" is that both the organizational administration and the employees/trainees have to face the realistic business problems through the solutions offered while they are "hooked in" a virtual platform-based training environment. Therefore, information technology managers play a crucial role since they facilitate the use of technological systems such as internet, intranet, video-conferences, satellite broadcasts etc., taking advantage of the most cost effective and time saving technological applications. The challenging aspect of corporate distance training is that the main core of participants, such as managers and high-level operators can be connected although geographically dispersed.

Another milestone over the new era in training is the so called e-learning based on "webbased instructions". During the last decades, we have experienced a revolution in training practices due to the development of web technology. The difference between traditional and web-based training is not related to the content of the knowledge itself. It deals with how knowledge is being contextualized. Web-based training courses are more effective in stimulating and maintaining learner's attention, given that the World Wide Web creates targeted, comprehensive and attractive educational material and means of delivering knowledge.

However, Beverly Abbey (Abbey 1999), from a pedagogical point of view, criticizes strongly the web-based instruction. Her main objection lies upon the issue of the validity of the information offered, due to the fact that the material is not always authenticated or signed by credible users in regard with reliable sources. She dramatically declares that the knowledge representation on the web is "...like the Swiss cheese, broad, thin and full of holes...". Another questionable issue is that the web-based hypertext environment is massive and excessively over-loaded, therefore, inexperienced users may be somehow "lost" or easily manipulated during their research.

\subsection{Knowledge management re-orientation in question}

A third milestone of the recent training developments deals with the transformation of education and training to strictly market and business-oriented courses. It looks like all modern colleges and universities prepare their students in order to directly enter into the world of business sectors and industries. Under this approach, on duty managers are taking the place of the old-fashion 
professors, while students are qualified according to their business skills and, therefore, training is actually a part of their business induction and career-path. G. Elliott and M. Crossley (Elliott and Crossley 1994), having empirically examined these concerns, concluded that the newly established pedagogical cultures are simply alternative models of management. Traditional lecturers usually oppose the idea of the majority of the academic foundations running as business industries or as the exclusive gateways to commercial services. The main reason is that such a prioritization disregard human studies, social responsibility and in general the needs of students as persons who most of all better off as sociable and in general educated personalities. It will be later in this paper discussed that crisis situations can be better controlled by crisis managers who exhibit a balance between expertise and encyclopedic, holistic as well as multi-faceted perspective of life values, ethos and ethics. Thus, modern training courses need to adopt a synthetic scope, further preparing students for complexity and radical global changes (Gilpin and Murphy 2008).

\subsection{Quality assurance issues}

At a systemic level, the educational tool drawing the scholars' attention is the quality assurance. Over the last years, quality assurance practices have been introduced thoroughly into several levels of training, regulating processes and procedures on the basis of relevant standards. According to $\mathrm{R}$. Brown (Brown 2004), the quality management thankfully has been transformed towards quality improvement rather than procedural accountability. There is a great difference between compliance with specific rules, on one hand, and quality management on the other, appealing to professional motivation and constructive assessments on how to better off as a training center.

The dimension of quality assurance mostly applicable in education fields is the involvement of external auditors, usually quality assurance agencies. They evaluate the performance of the training organizations and suggest improvements, being capable enough to make objective assessments, due to their expertise. It is true that especially the military organizations apply strict policies and internal auditing or self-assessments. However, as it was mentioned before, the accountability structures sometimes turn to be a dead end, since they are usually considered to be the one and only purpose of management. Therefore, considerable change and quality improvement can be guaranteed by external advisors that are not subject to biases coming out of any specific internal organizational cultures (Brown 2004).

\section{Particularities of Environments in Crisis}

\subsection{Phycological features of serving in combat}

In order to transfer the above mentioned modern training toolbox into the crisis management fields of training, we need to have an overall and complete view of the particularities that compose professional environments like those of the civil protection and the armed forces. Looking for the core characteristics of such rapid deployment civil and military units, T. W. Britt (Britt 2006) mentioned that a soldier, an officer or a rescue crew member differs from any other manager or employee since he/she is exposed to high risks and, therefore, may deal with human mortality. In simple words, it is the fear to face the natural but unanimously avoidable reality of a psychological stress, physical injury or even death; any action or reaction is inevitably affected by that fatal factor. The second particularity of those "in uniform" is the compelling presence of fatigue. Officers and soldiers along with rescuers are driven to the limits of their endurance and sometimes overpass them. Additionally, decision making, while performing preventive and/or corrective tasks against disasters, seems to be extremely tough since it is affected by lack of sleep, emotional stress multiplied the complexity of the environments and the overload of information. The outcome of these missions also depends on the morale of the fighters. The higher it is, the more likely they are capable to accomplish a mission, a disaster recovery and/or a rescue of human lives and properties. 
Technical Skills Crisis Matrix

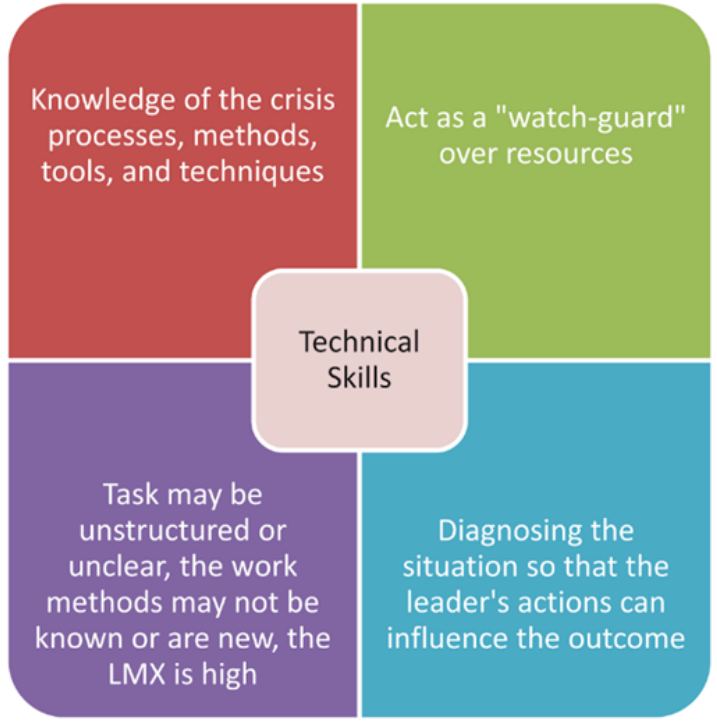

Fig. 1. Technical skills for crisis resolution (Powelson 2017)

Battle fields or natural disaster landscapes create havoc not only against team formations in general, but also to every single fighter; in other words, the personality of each crew member or his/her training level is more than worthy to be highly concerned (see Fig. 1 above). Inside the rescue and military units or their administrative headquarters involved, additional confusion may occur related to training deficiencies. Crisis management courses are a possible solution for strengthening the response capabilities and effectiveness. Paraskevas (Paraskevas 2006) states that the dynamically evolving character of the environments in crisis redefines Crisis Management Plans (CMPs) and training rules towards more innovative notions and sophisticated case studies like systemic analysis and chaos theory. CMPs are not automatically responsive, they need to have been "assimilated" into any specific organizational environment, taking into account colleagues' attitudes and educational attributes shaping an organization's resilience against critical threats and weaknesses (Baltos and Mitsopoulou 2007).

\subsection{Chaotic situations and professional response}

The involvement of risk management as well as systems theory in the study of business interruptions as well as natural, civil or military disasters introduces unpredictable variables that jeopardize the sustainability of services and operations in public or private sector (Piotrowski 2006). Given that a system by definition combines interdependent variables in order to achieve an objective, it should be clear that system factors may bring up exponentially increased results, in other words, the effects may surpass the causes in terms of impact and emergency (Senge 2006). The professional responders to a crisis, for instance the rescue teams, usually interact locally, facing difficulties and bottlenecks in the receipt and evaluation of critical information. Overloads of feedback may be possible, forcing receivers to re-evaluate and re-schedule according to the intensity and diversity of the incoming messages.

Lessons learned from disaster recovery and/or rescue operations refer by default to the main outlines of Chaos Theory, "where dysfunctional systems are considered a normal aspect of adaptation to high-stress conditions" (Piotrowski 2006). The term "crisis", having an ancient and classical origin, in Greek also means "judgement" (Paraskevas 2006). The resolution of crisis then 
requires crisis managers skilled enough and duly trained to think where there is no place or time for thinking. A crisis response system combined with proper training courses ideally provides recognition of all the critical situations, even before the crisis erupts. In hindsight, crisis management courses should emphasize on the mind's sharpness and agility of the leaders directing the crisis mitigation (see Fig. 2 below).

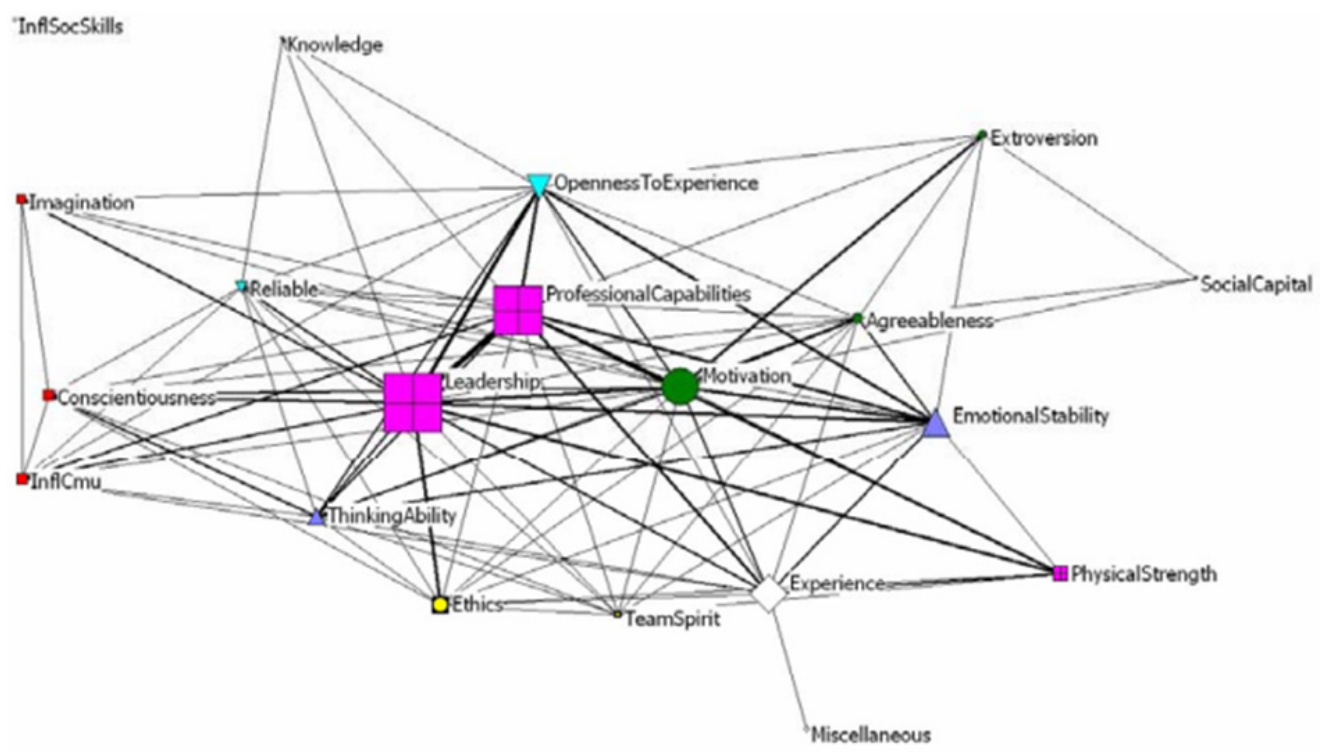

Fig. 2. Relations among skills and attributes scaled and preferred for the composition of crisis response teams (Baltos and Mitsopoulou 2007)

\section{Planning Crisis Management Trainings and Expected Results}

\subsection{A "ticking clock" speeds up all crisis situations}

Following the above described recent educational and training concepts, crisis management courses and military academies around the world have already adopted parts of the modernization process regarding recruiting specialists, teaching and supporting their students. The main concern of these institutions is to reinforce the programs with any recent technologies and best practices, aiming at training therein to be considered effective and competitive. It is also a fact that civil protection and armed forces accomplish continuous and intense learning programs for their staff that have already been tested in a long list of real emergency situations on numerable levels of human activity and survival, e.g. earthquakes, floods, famines, chemical attacks, nuclear and radiation accidents, terrorist attacks, cyber-attacks, energy supply disruptions, banking breakdowns, wildfires, anti-terror campaigns, hurricanes, etc. proven in general terms improved in comparison to similar past incidents.

However, this variety of engagements should be organized in a more permanent and detailed way through the objectives of a general educational context and vision. For example, post-graduate studies should systematically include crisis management disciplines, in order to ensure a continuing stream of skilled and talented employees in the organizations involved. The scope of such operations is so deep and wide that we could even claim the dawn of a new science or at least a scientific methodology, researching the impact and the likelihood of abnormal and unexpected factors as well as the avoidance or containment of the uncertainty.

By tradition, the military training is a highly and strictly structured paradigm, as long as it attempts to 
ensure a measure of order recovery and situational predictability. Therefore, it relies upon clear-cut procedures and protocols, mainly operating in bureaucratic environments. On the opposite side, many analysts, such as M. J. Cook, J.N. Noyes and Y. Masakowski (Noyes, Cook, and Masakowski 2012), have commented that the predominance of bureaucratic procedures, strict protocols and apriori segregation of duties has formed managerial behaviors that, due to lack of flexibility, agility and adaptability, are not capable to lead them to a proper and resourceful decision-making.

For example, a well-known situation where the military formations need to be familiar with factors like novelty, surprise and improvisation is the natural disaster relief missions. Under such conditions formal hierarchical protocols have been proven to be ineffective. The relief comes from light team formations with cross functional structure and on-site situational awareness. This is why the anticipation of and readiness to control any potential risk should be the starting point in the respective training centers. E Salas and G.A. Klein (Salas and Klein 2001) highlighted the importance of training the officers at improvising and increasing the range of their responsiveness repertoire.

Another positive fact is that national authorities, international and non-governmental organizations, since a long time ago, have adopted plenty of bottom-up training programs, whereas the already highly trained may participate as trainers in the training of other less experienced, transferring directly their knowledge and expertise. However, these processes should be enriched with additional academic staff from major research institutions, towards the establishment of joint and interdisciplinary educational programs in collaboration with scholars and practitioners willing to exchange ideas, best practices and lessons learned.

The ideal should be the balance among academic courses, "on the job" training and hightechnology applications. Therefore, we should agree with the proposal of the US NAVY Committee on Technology for Future Naval Forces (CTFN et al. 1997), according which "...we have to invest more in the conversion of the conventional forms of training to technology-based and technology distributed training programs....". The already existing platforms for the web-based distance learning that operate at the moment simply as pilot-programs should be carefully expanded and integrated to the rest of the existing training platforms, in order to meet the needs of the modern training systems.

Among the most effective and efficient training tools, the simulation training devices have been adopted by all advanced armed forces, while they are advisable in regard with all the range of trainings for emergencies. The simulation models are based on creating virtual reality environments. They create virtual crisis realities, activate users' attention, imagination, cognition and responsiveness. Thus, the trainees improve their judgment and ability to map the situations and make proper decisions out of virtual worst cases scenarios. According to R. Thierauf (Thierauf 2001) it is fully worthy to invest in acquisition and operation of simulation projects. The generation of interactive on-screen environments, involving iconic stress tests as well as other hostile and/or friendly players, allows civil and military staff to learn from making virtual but not fatal mistakes, resulting into decreased mistakes and minimum possible casualties during the "real-time operations".

\subsection{Activating the emotional intelligence and team work leverage}

It is a fact that in the professional and in vivo operations the element of collaboration and team work is emphatically present, affecting positively the results of the total performance. Under these circumstances, it is obvious that safety and security operators should elaborate training patterns compatible to what T. W. Britt and A. B. Adler (Britt and Adler 2003) described as "comradeship". He highlighted the importance of this co-working issue in selecting and positioning team members in effective formations, repeating the famous saying: "four brave ones, who do not know each other will not dare to attack a lion. Four less brave, but trusting well each other, being sure they will be mutually supported by all means, will attack resolutely". 
Emotional Intelligence Crisis Matrix

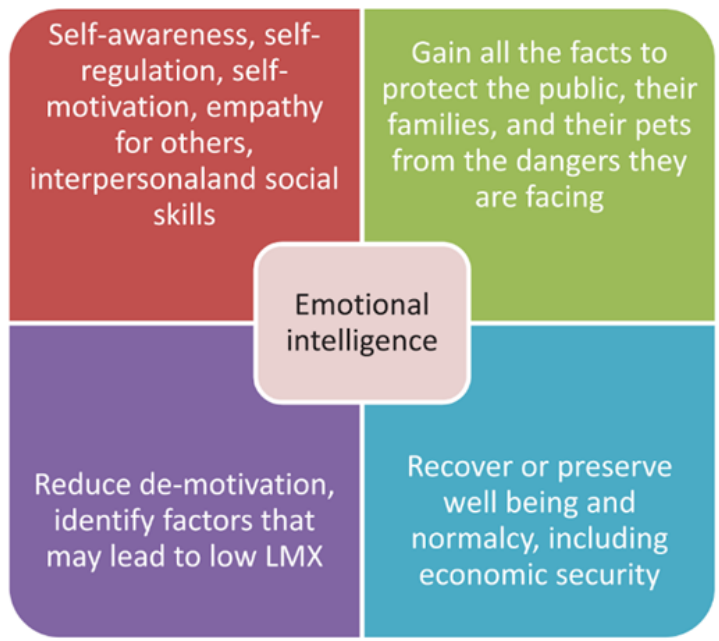

Fig. 3. Emotional intelligence elements for crisis resolution (Powelson 2017)

Therefore, it seems as an emergency to enhance training courses with all the modern training tools that stimulate and develop ethics, collaborative spirit, responsibility bonds and professional cooperation. When a system relies on bureaucracy and only, may operate under normal circumstances, but when uncertainty and risks prevail, there is a great possibility for this system to break down and collapse. Basic shortfalls that may be created due to severe bureaucracy include communication and coordination distortions throughout the operations. N. Kapucu (Kapucu 2006) sums up the importance of effective communication and training against crises, highlighting timely sharing of valid information, reciprocity and mutual trust in decentralised team formations (see Fig. 3 above).

\section{3 "Anti-crisis leadership" - Intuition is the source of powers}

Flexibility and adaptability should be primarily reflected into the training plans and vision (Fink 1992). Training courses should always include and emphasize on emergencies and risk management. According to Erik Hollnagel (Hollnagel 2003), there is a series of requirements to be fulfilled in order to swift the training patterns towards increased readiness and prepared-ness under high stress situations:

- The first requirement is related to the functionalism theory, according to which academies and colleges suggest functional and cognitive engineering schemes that describe literally how to react when specified problems suddenly appeared and should be immediately faced.

- The second one is the prompt use of expertise. The crisis managers need to be experts on offering solutions and advices, in other words, they need to be highly aware of certain situations up to the point that they somehow respond "automatically" based on their inner tacit knowledge and empirical awareness.

- The third requirement, which is closely related to the first one, is described as "cognitive architectures". In a few words, crisis managers should be trained in mapping the situations, scanning the factors that rule the outcome of the activities taken, especially when those factors are changing under conditions that could be considered threatening, even for human lives.

- The fourth one deals with what was described as "ethnography of workplaces". The 
training courses should somehow simulate the workplace environment. Taking into account the particularities of the military and/or civil protection "workplace" area, we could imagine how crucial it is for a crisis manager to be able to analyze the conditions' changes and the relevant risks unexpectedly appeared, focusing on the cultural parameters that explain actions and reactions, foreseeing the next forthcoming problems.

- The fifth requirement is the so called "naturalistic" decision making. According to that theory, crisis management students should learn decision making techniques that solve complicated problems. The naturalistic aspect of the afore-mentioned concept is related to the fact that students learn how to react in a natural way, sometimes driven by intuition rather than rationality.

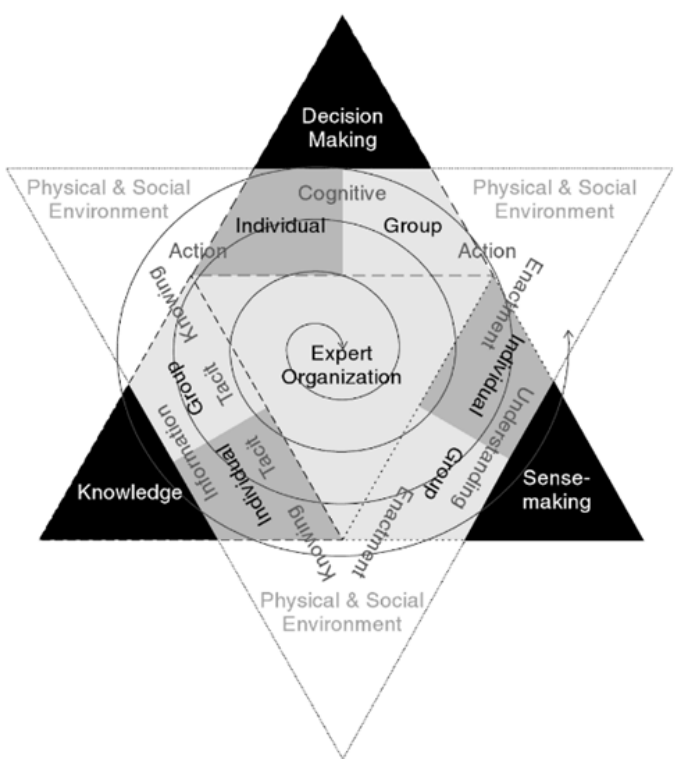

Fig. 4. The "Expert Organization" (Gilpin and Murphy 2008)

"Anti-crisis leadership" should demonstrate availability of a crisis culture (Mitroff and Alpaslan 2003). On that basis (see Fig. 4 above) of the multiple emergencies' particularities, the designers of relevant training courses have to conduct a job/task analysis anytime they launch a new academic course or training exercise. We could even say that the so-called job/task analysis is an essential pre-training element, affecting the overall success of the training. T. W. Britt, A. B. Adler and C. A. Castro (Britt, Adler, and Castro 2006) expanded the application of job/task analysis as a pertaining element, including in the job/task analysis not only the factor of detailing the specifications of the tasks, but also emphasizing on the competencies needed for each specific task, for example, communication, adaptability, co-working experience, etc. Given that the crisis setting changes through unpredictable situations, the basic job/task analysis needs to be upgraded to the so-called cognitive task analysis (CTA). The traditional setting of job/task descriptions should also be related to assignments that vary not only for each person but also for each one of the next critical seconds. The personality analysis or the situation awareness support an effective cognition over the dynamically changing environments, therefore, any task analysis or relevant training should encompass them, in order to provide first aid officers and rescuers with the adaptability needed to survive over really extreme and adverse environments. Intuition is the source of power, according to Klein (Klein 1999), not as metaphysical talent or encyclopedic thesaurus, but as a quick and "smart" response due to multiple training and accumulated experience. 


\section{Conclusions and Policy Implications}

The three-fold of knowledge, skills, and ability (KSA) is significant, as it is widely accepted, for the effectiveness of a formation, but it is not enough on its own to transform the members of a group into an effective team (Athanasaw 2003). The catalyst for individual and collective excellence is the inclusion in the team of highly experienced members capable to conceptualize collaborative coworking much more intuitively than lower experienced team members; under the dynamically changing and threatening conditions of crisis management operations, leaders should visualize the "big picture" of the operational theatre (Rentsch, Heffner, and Duffy 1994). Notions and values like alerted-ness, shared-ness of information, cultural awareness combined with insightful leadership and stress management may determine the success or failure of actions taken (Baltos and Mitsopoulou 2007). Planning for crisis management includes student-centric training, networking practice in a way that all actors collaborate organically as parts of an interoperable dynamic system (Gibbons 2007). Simulations, exercises and cognition task analyses then show the way of safety and security for the societies to achieve stability, peace and prosperity.

\section{References}

Abbey, B., (2000). Instructional and cognitive impacts of Web-based education. London: Idea Group Inc (IGI).

Athanasaw, Y. (2003). Team Characteristics and Team Member Knowledge, Skills, and Ability Relationships to the Effectiveness of Cross-Functional Teams in the Public Sector. International Journal of Public Administration, 26, 1165.

Baltos, G., and Mitsopoulou, Z. (2007). Team formation under normal versus crisis situations: leaders' assessments of task requirements and selection of team members. MBA Thesis. [Online] Available: http://www.dtic.mil/dtic/tr/fulltext/u2/a473289.pdf (23 April, 2018)

Britt, T. W. (2006). Military Performance. Washington: Greenwood Publishing Group.

Britt, T, W., Adler, A, B., \& Castro, C. A. (2006). Military life: the psychology of serving in peace and combat. Washington: Greenwood Publishing Group.

Brown, R. (2004). Quality assurance in higher education: the UK experience since 1992. Routledge.

Committee on Technology for Future Naval Forces. (1997). Technology for the United States Navy and Marine Corps, 2000-2035 - Becoming a 21st Century Force: Becoming a 21st-Century Force: Human Resources. Bristol: The National Academies Press.

Gibbons, D. E. (2007). Communicable crises: prevention, response, and recovery in the global arena. IAP.

Cook M. J., Noyes J. M., \& Masakowski Y. (2007). Decision making in complex environments. Bristol: Ashgate Publishing, Ltd.

Elliott, G., and Crossley, M. (1994). Qualitative research, educational management and the incorporation of the further education sector. Educational Management \& Administration, 22 (3), 188-197.

Fink, S., L. (1992). High commitment workplaces. Greenwood Publishing Group.

Gilpin, D. R., and Murphy, P. J. (2008). Crisis Management in a Complex World. Oxford University Press.

Haddow, G., \& Bullock, J. (2005). Introduction to Emergency Management (2nd edition ed.). ButterworthHeinemann.

Hollnagel, E. (2003). Handbook of cognitive task design. London: Lawrence Erlbaum Associates.

Khosrow-Pour, M., (2002). Web-based instructional learning. Newcastle upon Tayne: Idea Group Inc (IGI)

Kapucu, N. (2006). Interagency communication networks during emergencies: Boundary spanners in multiagency coordination. The American Review of Public Administration, 36 (2), 207-225.

Klein, G. (1999). Sources of Power: How People Make Decisions. Boston: The MIT Press.

Mitroff, I., and Alpaslan, M. A. (2003). Preparing for evil. Harvard Business School Publications.

Paraskevas, A. (2006). Crisis management or crisis response system? A complexity science approach to organizational crises. Management Decision, 44, 892-907.

Piotrowski, C. (2006). Hurricane Katrina and Organization Development: Part 1. Implications of Chaos Theory.

Organization Development Journal, 24, 10-19.

Powelson, M. (2017). Hurricane Katrina and the Lessons of Disaster Relief. Cambridge Scholars Publishing.

Rentsch, J., R., Heffner, T., S., \& Duffy, L. T. (1994). What You Know Is What You Get From Experience: Team Experience Related to Teamwork Schemas. Group \& Organization Management, 19, 450-474.

Salas, E., \& Klein, G. A. (2001). Linking expertise and naturalistic decision making. New York: Lawrence Erlbaum Associates.

Senge, P. M. (2006). The Fifth Discipline: The Art \& Practice of the Learning Organization. London: Currency. Thierauf, R, J. (2001). Effective business intelligence systems. Greenwood Publishing Group. 